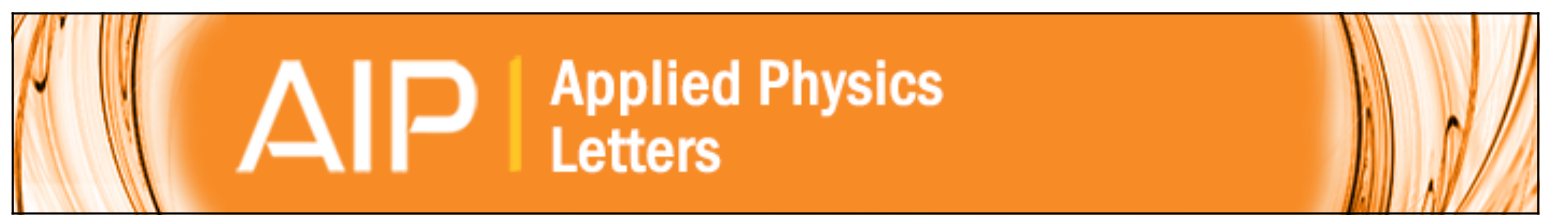

\title{
Electrical characterization of the soft breakdown failure mode in $\mathrm{MgO}$ layers
}

E. Miranda, E. O'Connor, K. Cherkaoui, S. Monaghan, R. Long, D. O'Connell, P. K. Hurley, G. Hughes, and P. Casey

Citation: Applied Physics Letters 95, 012901 (2009); doi: 10.1063/1.3167827

View online: $\mathrm{http} / / / \mathrm{dx}$. doi.org/10.1063/1.3167827

View Table of Contents: http://scitation.aip.org/content/aip/journal/apl/95/1?ver=pdfcov

Published by the AIP Publishing

Alp Re-register for Table of Content Alerts

\section{Create a profile.}




\title{
Electrical characterization of the soft breakdown failure mode in MgO layers
}

\author{
E. Miranda, ${ }^{1, a)}$ E. O'Connor ${ }^{2}{ }^{2}$ K. Cherkaoui, ${ }^{2}$ S. Monaghan, ${ }^{2}$ R. Long, ${ }^{2}$ D. O'Connell, ${ }^{2}$ \\ P. K. Hurley, ${ }^{2}$ G. Hughes, ${ }^{3}$ and P. Casey ${ }^{3}$ \\ ${ }^{1}$ Escola Tècnica Superior d'Enginyeria, Universitat Autònoma de Barcelona, Campus UAB, 08193 \\ Bellaterra, Barcelona, Spain \\ ${ }^{2}$ Tyndall National Institute, University College Cork, Cork, Ireland \\ ${ }^{3}$ School of Physical Sciences, Dublin City University, Glasnevin, Dublin 9, Ireland
}

(Received 21 April 2009; accepted 2 June 2009; published online 7 July 2009)

\begin{abstract}
The soft breakdown (SBD) failure mode in $20 \mathrm{~nm}$ thick $\mathrm{MgO}$ dielectric layers grown on $\mathrm{Si}$ substrates was investigated. We show that during a constant voltage stress, charge trapping and progressive breakdown coexist, and that the degradation dynamics is captured by a power-law time dependence. We also show that the SBD current-voltage $(I-V)$ characteristics follow the power-law model $I=a V^{b}$ typical of this conduction mechanism but in a wider voltage window than the one reported in the past for $\mathrm{SiO}_{2}$. The relationship between the magnitude of the current and the normalized differential conductance was analyzed. () 2009 American Institute of Physics.
\end{abstract}

[DOI: $10.1063 / 1.3167827]$

In recent papers, magnesium oxide $(\mathrm{MgO})$ has been pointed out as an appealing material for use as a gate insulator in metal-oxide-semiconductor (MOS) devices both for $\operatorname{low}^{1}$ and high $^{2}$ power applications. The main features of this alkaline earth oxide are: a large band gap in the range $7.3 \mathrm{eV}$ (Ref. 1)-7.8 eV (Ref. 3), ensuring sufficiently large band offsets with $\mathrm{Si}$ and therefore low leakage current, a dielectric permittivity $\kappa$ ranging from 6.7 (Ref. 4) to 10 (Ref. 3) depending on the preparation method, high thermal conductivity suitable for large power dissipation, ${ }^{2}$ and significantly, its chemical inertness, which minimizes the formation of an interfacial layer when deposited on $\mathrm{Si}$ substrates. ${ }^{1}$ In this letter, we have focused attention on a specific reliability aspect of $\mathrm{MgO}-\mathrm{Si}$ system, which is the conduction mechanism after the occurrence of soft breakdown (SBD) events. ${ }^{5}$ As is commonly accepted, this failure mode arises as a consequence of the formation of a single or multiple nanoconstrictions across the oxide layer after reaching a certain threshold density of defects caused by the electrical stress. This is a localized conduction mode, independent of the device area, and has been reported to occur in a number of high- $\kappa$ thin films other than $\mathrm{SiO}_{2}{ }^{6}$ However, contrary to the widespread opinion that this failure mechanism can only be observed in ultrathin $\left(t_{\mathrm{ox}}<6 \mathrm{~nm}\right)$ oxides, we have detected it in $\mathrm{MgO}$ films with nominal $t_{\mathrm{ox}}=20 \mathrm{~nm}$, with $t_{\mathrm{ox}}$ the oxide thickness. Notably, our sample thickness almost doubles the maximum $\mathrm{SiO}_{2}$ thickness $\left(t_{\mathrm{ox}}=12 \mathrm{~nm}\right)$ for which SBD has been reported. ${ }^{7}$ In order to demonstrate that we are strictly dealing with the same charge transport mechanism, we have carried out a thorough analysis of the conduction characteristics starting with the widely accepted power-law model $I=a V^{b}$, where $a$ and $b$ are constants. ${ }^{8}$ This allows us to examine the limits of the validity of the referred model, identify SBD events with leakage current levels several orders of magnitude lower than those detected in the past, which in thinner oxides would have been overlooked and, finally, extend the relationship

\footnotetext{
a) Author to whom correspondence should be addressed. Tel.: 34-935813183. FAX: 34-93-5812600. Electronic mail: enrique.miranda@uab.es.
}

exhibited by the fitting parameters $a$ ( $I$ at $1 \mathrm{~V})$, and $b$ $(=d \ln I / d \ln V)$ previously reported for the $\mathrm{SiO}_{2} / \mathrm{Si}$ system.

Hydrogen terminated Si surfaces were prepared by dipping $n$ - and $p$-type (100) Si wafers $\left(10^{15} \mathrm{~cm}^{-3}\right)$ in a solution of 5\% hydrofluoric acid (HF) for $1 \mathrm{~min}$, followed by rinsing in de-ionized water, and dried using nitrogen and were then immediately loaded into the deposition system. $\mathrm{MgO}$ films of nominal $t_{\mathrm{ox}}=20 \mathrm{~nm}$ with $\kappa \sim 8.1$ (equivalent oxide thickness $\mathrm{EOT} \approx 9.5 \mathrm{~nm}$ ) were deposited by electron beam evaporation from $99.9 \% \mathrm{MgO}$ pellets at a rate of $0.2 \AA / \mathrm{s}$, at $180{ }^{\circ} \mathrm{C}$. The samples were capped in situ with $100 \mathrm{~nm}$ of amorphous silicon $(\alpha-\mathrm{Si})$ using a second e-beam source. For the $\mathrm{NiSi}$ gate process, nickel was deposited by electron beam evaporation $(\sim 80 \mathrm{~nm})$ through a patterned resist mask followed by a lift-off process. The rapid thermal annealing is a one step process at $500{ }^{\circ} \mathrm{C}$ for $30 \mathrm{~s}$ in $\mathrm{N}_{2}$. The area of the devices tested is $1.6 \times 10^{-5} \mathrm{~cm}^{2}$. Details about the $\mathrm{SiO}_{2}$ samples with $t_{\mathrm{ox}} \approx 4 \mathrm{~nm}$ considered in this study can be found in Ref. 5 .

The dielectric breakdown of the $\mathrm{MgO}$ layer can be induced by either ramped or constant voltage stresses. Figure 1 shows the effects of the latter type of stress on the leakage

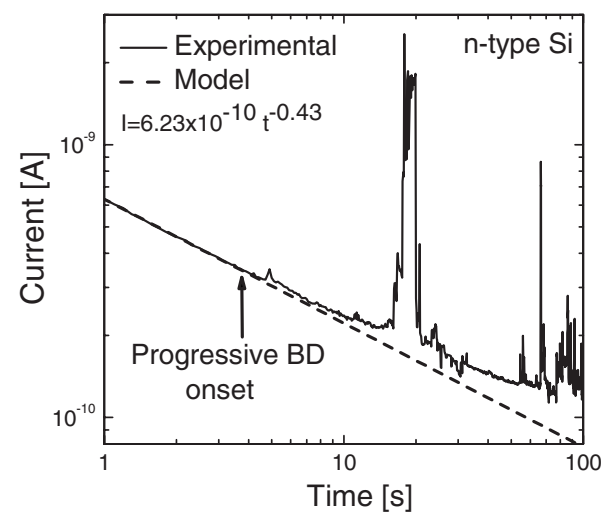

FIG. 1. Evolution of the gate leakage current under a constant voltage stress at $8 \mathrm{~V}$. The solid and dashed lines correspond to experimental data and fitting results, respectively. 


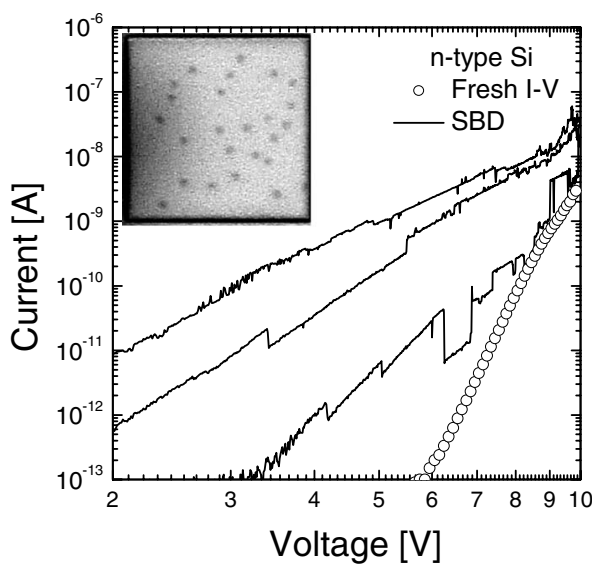

FIG. 2. Experimental current-voltage characteristics. Symbols and lines correspond to the fresh and SBD $I-V$ characteristics, respectively. Notice the use of log-log axis in order to emphasize the power-law dependence. The maximum voltage stress is $10 \mathrm{~V}$. The inset shows several SBD spots distributed over the device area.

current. Notice that the current decreases according to the empirical law $I(t)=I_{0} t^{-\alpha}$, where $I_{0}$ and $\alpha$ are constants and $t$ is the stress time, in agreement to what has been observed in other high- $\kappa$ s such as $\mathrm{Al}_{2} \mathrm{O}_{3}$ and $\mathrm{HfO}_{2} .{ }^{9}$ The origin of the current decay is still unclear but, since the process is partially reversible, it might be related to transient and permanent charge trapping effects in pre-existing and induced traps. The onset of dielectric breakdown is detected as a noise increment in the $I-t$ characteristic and this event is followed by a progressive increase of the current [progressive breakdown (PBD)]. Alternatively, SBD can be achieved by successive ramped voltage stresses as shown in Fig. 2. The major damage to the sample is caused at the end of each sweep and, in most of the cases, no current jump associated with SBD is observed, an indication of the progressive opening of the leakage spots. The switching behavior, typical of digital SBD conduction, ${ }^{10}$ is clearly observed in the second and third $I-V$ characteristics. Contrary to what happens with ultrathin $\mathrm{SiO}_{2}$ layers, in which SBD is only observable in a very limited voltage range because of the tunneling current that flows through the nondamaged device area, ${ }^{11}$ in the present case of a $20 \mathrm{~nm}$ thick sample the SBD curve can be detected in a larger voltage window. The SBD $I-V$ curves cannot be associated with a single constriction and it is more likely that many leakage sites contribute to the total current. The inset in Fig. 2 supports this assertion and shows the occurrence of several breakdown spots on the gate electrode after the stress sequence. According to our data the breakdown field for $\mathrm{SBD}$ in $\mathrm{MgO}$ is around 5-6 $\mathrm{MV} / \mathrm{cm}$ which is comparable to the breakdown field in $\mathrm{HfO}_{2}(4-5 \mathrm{MV} / \mathrm{cm})$ but much lower than in $\mathrm{SiO}_{2}(13-14 \mathrm{MV} / \mathrm{cm}) .{ }^{12}$ Regarding the voltage dependence of the SBD curves, notice that the linear relationship between $\log (I)$ and $\log (V)$ supports the use of the power-law model. From the physical viewpoint, this model has been related to the conduction properties of nonlinear resistances networks with a distribution of percolation thresholds. ${ }^{13}$ However, it is worth pointing out that the model is unable to capture the experimental fact that for larger damaged areas the slope of the $I-V$ curves becomes shallower. This trend was first observed by Okada and Taniguchi ${ }^{14}$ in $\mathrm{SiO}_{2}$ and was thoroughly analyzed in Refs. 8 and 15 for samples subjected to electrical stress and heavy ion irradia-

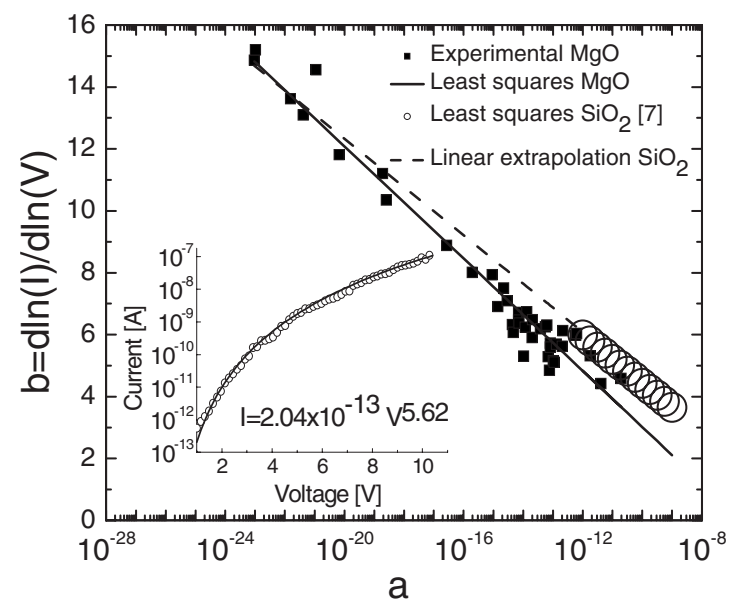

FIG. 3. Relationship between the parameters $a$ and $b$ in the power-law model. The circles correspond to the region of $\mathrm{SiO}_{2}\left(\mathrm{t}_{\mathrm{ox}} \approx 3.8-4.3 \mathrm{~nm}\right)$ experimental data given by the equation $b=-0.78 \log (a)-3.27$ [8]. The squares correspond to the parameters extracted from the MgO SBD $I-V$ curves. The solid line is given by the equation $b=-0.90 \log (a)-6.05$.

tion, respectively. The change in slope is consistent with the fact that the hard breakdown (HBD) $I-V$ characteristic has slope close to unity, ${ }^{5}$ as expected for a simple resistor. This relationship between the magnitude of the current $a$ and its normalized differential conductance $b$ which has not been reported before for a high- $\kappa$ with such a large thickness, has been explained in terms of the mesoscopic transport properties of the breakdown paths. ${ }^{16}$ Figure 3 shows the correlation between $a$ and $b$ obtained from a large number of $I-V$ curves and the inset shows a typical fitting to a SBD $I-V$ characteristic in $\mathrm{MgO}$ using the power-law model. The points in Fig. 3 cannot be considered as a statistically independent data ensemble since some of them were extracted from $I-V$ s measured in the same sample after further degradation. On the contrary, in Ref. 8 the parameters were extracted from independent measurements. Even though this parameter extraction procedure can lead to uncertainty, what is remarkable is that both data sets $\left(\mathrm{MgO}\right.$ and $\left.\mathrm{SiO}_{2}\right)$ follow very similar linear trends. It is worth mentioning that thicker $\mathrm{SiO}_{2}$ films than those considered in this study only exhibit the HBD mode so that the comparison between samples with similar large thicknesses is not possible. Figure 3 also shows that the correlation between $a$ and $b$ in $\mathrm{MgO}$ covers nearly ten orders of magnitude of the parameter $a$, whereas for $\mathrm{SiO}_{2}$ this correlation holds in a narrower range. A possible interpretation for the observed correlation is that the size of the spots is strongly linked to its conducting properties, in such a way that the damaged area does not enter as a simple prefactor in the current expression as commonly assumed for standard transport mechanisms such as tunneling, Poole-Frenkel or Schottky emission. In addition, Fig. 3 shows that many of the SBD events occurring in $\mathrm{MgO}$ are softer than those detected in $\mathrm{SiO}_{2}$. This can be a matter of the sample thickness, where the magnitude of tunneling currents permits the observation of softer events, but the possibility that it is related to particular features of the conducting spots, such as the microscopic arrangement of the defect sites, cannot be ruled out. In addition, the fact that SBD can be observed in a thick oxide layer is compatible with the idea that what fundamentally governs the conduction characteristics is the bottleneck of the constriction and not its total dength. 16 


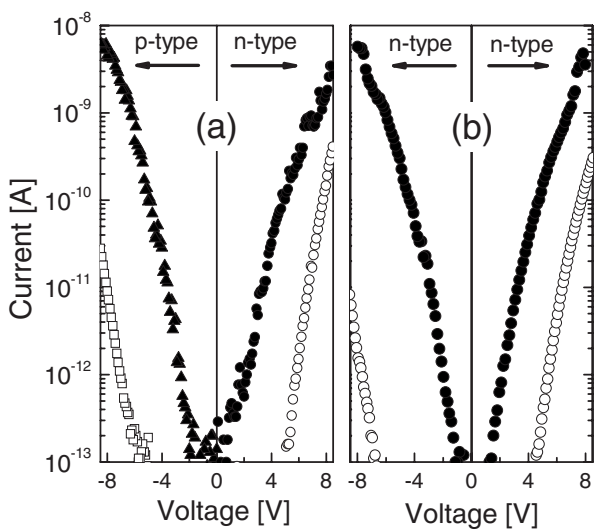

FIG. 4. (a) Effect of the substrate type and (b) effect of the injection polarity, on the SBD current. Open and filled symbols correspond to the fresh and SBD $I-V$ characteristics, respectively.

To complete the electrical characterization of SBD in $\mathrm{MgO}$, the roles played by the substrate type and gate voltage $\left(V_{G}\right)$ polarity were investigated. Figure $4($ a) shows that similar SBD currents can be found both for $\mathrm{n}\left(V_{G}>0\right)$ and $p$-type $\left(V_{G}>0\right)$ substrate samples in accumulation conditions. Identical results arise for a $n$-type $\mathrm{Si}$ sample biased with both injection polarities as shown in Fig. 4(b). Notice that this is not the case for the fresh $I-V \mathrm{~s}$. These plots indicate that the injecting electrode does not play any major role after SBD as long as there is sufficient charge available for conduction. The current seems to be limited by the breakdown path itself, so that its transmission properties become a key element for the description of the phenomenon. ${ }^{5}$

In summary, the postbreakdown electrical behavior of $\mathrm{MgO}$ layers in MOS structures was investigated. It was shown that SBD conduction can be detected in a relatively thick high- $\kappa$ film and that the connection between the charge transport mechanisms in $\mathrm{MgO}$ and $\mathrm{SiO}_{2}$ goes beyond the fact that they share the same power-law $I-V$ model. The correlation found between the model parameters in both of the materials investigated, and consequently, the apparent simplicity of the underlying physics, should perhaps be regarded as an indication of the low dimensionality of these conducting structures.

E.M. acknowledges the Generalitat de Catalunya (BE2007), the Ministerio de Ciencia y Tecnología (MCyT), Spain (Grant No. TEC2006-13731-C02-01) and the Science Foundation Ireland (SFI) under the Walton Awards scheme (Grant No. 07/W.1/I1828) for their financial support. All authors acknowledge the SFI grant (Grant No. 05/IN/1751) and the SFI Ireland National Access Program at the Tyndall National Institute.

${ }^{1}$ L. Yan, C. Lopez, R. Shrestha, E. Irene, A. Suvorova, and M. Saunders, Appl. Phys. Lett. 88, 142901 (2006).

${ }^{2}$ A. Posadas, F. Walker, C. Ahn, T. Goodrich, Z. Cai, and K. Ziemer, Appl. Phys. Lett. 92, 233511 (2008).

${ }^{3}$ II-VI and I-VII Compounds; Semimagnetic Compounds, LandoltBörnstein, New Series, Group III Vol. 41B, edited by O. Madelung (Springer, Berlin, 1999).

${ }^{4}$ B. Brennan, S. Mc Donnell, and G. Hughes, J. Phys.: Conf. Ser. 100, 042047 (2008).

${ }^{5}$ E. Miranda and J. Suñé, Microelectron. Reliab. 44, 1 (2004).

${ }^{6}$ G. Ribes, J. Mitard, M. Denais, S. Bruyere, F. Monsieur, C. Parthasarathy, E. Vincent, and G. Ghibaudo, IEEE Trans. Device Mater. Reliab. 5, 5 (2005)

${ }^{7}$ S. Lombardo, A. La Magna, C. Gerardi, M. Alessandri, and F. Crupi, Appl. Phys. Lett. 75, 1161 (1999).

${ }^{8}$ E. Miranda, J. Suñé, R. Rodríguez, M. Nafría, and X. Aymerich, IEEE Electron Device Lett. 20, 265 (1999).

${ }^{9}$ S. Zafar, A. Callegari, E. Gusev, and M. Fischetti, J. Appl. Phys. 93, 9298 (2003).

${ }^{10}$ T. Tomita, H. Utsunomiya, T. Sakura, Y. Kamakura, and K. Taniguchi, IEEE Trans. Electron Devices 46, 159 (1999).

${ }^{11}$ E. Miranda, J. Suñé, R. Rodríguez, M. Nafría, and X. Aymerich, SolidState Electron. 43, 1801 (1999).

${ }^{12}$ J. McPherson, J. Kim, A. Shanware, H. Mogul, and J. Rodríguez, Proceedings of the IEEE International Electron Devices Meeting IEDM'02 (IEEE, New York, 2002), p. 633.

${ }^{13}$ M. Houssa, T. Nigam, P. W. Mertens, and M. M. Heyns, J. Appl. Phys. 84, 4351 (1998).

${ }^{14}$ K. Okada and K. Taniguchi, Appl. Phys. Lett. 70, 351 (1997).

${ }^{15}$ A. Cester, J. Suñé, and E. Miranda, Appl. Phys. Lett. 79, 1336 (2001).

${ }^{16}$ J. Suñé and E. Miranda, Tech. Dig. - Int. Electron Devices Meet. 2000, 533. 